\title{
LPS injection reprograms the expression and the 3' UTR of a CAP gene by alternative polyadenylation and the formation of a GAIT element in Ciona intestinalis
}

\author{
Aiti Vizzini ${ }^{\mathrm{a}, 1}$, Angela Bonura ${ }^{\mathrm{b}, 1}$, Valeria Longo ${ }^{\mathrm{b}}$, Maria Antonietta Sanfratello ${ }^{\mathrm{a}}$, \\ Daniela Parrinello ${ }^{a}$, Matteo Cammarata ${ }^{\mathrm{a}}$, Paolo Colombo ${ }^{\mathrm{b}, *}$ \\ a Dipartimento di Scienze e Tecnologie Biologiche, Chimiche e Farmaceutiche, Via Archirafi 18, Palermo, Italy \\ ${ }^{\mathrm{b}}$ Istituto di Biomedicina ed Immunologia Molecolare “Alberto Monroy" del Consiglio Nazionale delle Ricerche, Via Ugo La Malfa 153, Palermo, Italy
}

\section{A R T I C L E I N F O}

\section{Article history:}

Received 28 June 2016

Received in revised form 3 August 2016

Accepted 4 August 2016

Available online 8 August 2016

\section{Keywords:}

Ciona intestinalis

CAP protein

Polyadenylation

GAIT element

LPS

\begin{abstract}
A B S T R A C T
The diversification of cellular functions is one of the major characteristics of multicellular organisms which allow cells to modulate their gene expression, leading to the formation of transcripts and proteins with different functions and concentrations in response to different stimuli. CAP genes represent a widespread family of proteins belonging to the cysteine-rich secretory protein, antigen 5 and pathogenesis-related 1 superfamily which, it has been proposed, play key roles in the infection process and the modulation of immune responses in host animals. The ascidian Ciona intestinalis represents a group of proto-chordates with an exclusively innate immune system that has been widely studied in the field of comparative and developmental immunology. Using this biological system, we describe the identification of a novel APA mechanism by which an intronic polyadenylation signal is activated by LPS injection, leading to the formation of a shorter CAP mRNA capable of expressing the first CAP exon plus 19 amino acid residues whose sequence is contained within the first intron of the annotated gene. Furthermore, such an APA event causes the expression of a translational controlling cis-acting GAIT element which is not present in the previously isolated CAP isoform and identified in the 3'-UTR of other immunerelated genes, suggesting an intriguing scenario in which both transcriptional and post-transcriptional control mechanisms are involved in the activation of the CAP gene during inflammatory response in $C$. intestinalis.
\end{abstract}

(c) 2016 Elsevier Ltd. All rights reserved.

\section{Introduction}

One of the hallmarks of eukaryotic genes is the presence of intervening sequences within protein coding information. The differential inclusion of exons and introns or portions of them in mature mRNAs results in the possibility of expressing multiple transcripts with different coding capacities from the same single gene (de Klerk and t Hoen, 2015). In this way, cells can enormously expand their flexibility, modulating their activities and displaying dynamic changes in response to independent stimuli (Anderson, 2010). Several studies have revealed how alternative splicing and alternative polyadenylation (APA) are exceedingly common events that occur throughout innate immunity and fine-tune almost all

\footnotetext{
* Corresponding author at: Istituto di Biomedicina ed Immunologia Molecolare “Alberto Monroy" Via Ugo La Malfa 153, 90146 Palermo, Italy.

E-mail address: paolo.colombo@ibim.cnr.it (P. Colombo).

1 These authors contributed equally to this work.
}

steps in the process. In this respect, immune genes are coordinately and temporally regulated in response to distinct stimuli. Transcriptional and post-transcriptional mechanisms that modify mRNA expression, stability and/or translation provide a rapid and flexible control of this process and are particularly important in coordinating the initiation and resolution of inflammation (Anderson, 2010).

The use of APA sites represents a regulatory level by which cells can generate different protein isoforms with different functions or with mRNAs differing in the length of their $3^{\prime}$ untranslated regions (UTR) (Batra et al., 2015). APA mechanisms can be divided into two major types: the simplest and more frequent are named UTRAPA(UnTranslated Region-Alternative PolyAdenylation) where the alternative $\operatorname{poly}(\mathrm{A})$ sites are located in the $3^{\prime}$ UTR of the mRNAs, with the majority of them residing closer to the stop codon (proximal) compared to canonical pA sites (distal). This kind of alternative mechanism results in the shortening of the $3^{\prime}$ UTR without changing coding capacity. Through the alteration of the $3^{\prime}$ UTRs, APA 
potentially regulates the stability, cellular localization and translation efficacy of target RNAs as 3' UTR serve as binding regions for factors that control these regulatory layers (i.e. micro RNAs and RNA binding proteins). Furthermore, 3'UTR of the mRNA of pro-inflammatory proteins can contain regulatory elements such as Interferon- $\boldsymbol{\gamma}$-activated inhibitor of translation (GAIT) that direct their degradation and/or translational repression (Fox, 2015).

On the other hand, CR-APA (Coding Region-Alternative PolyAdenylation) is a mechanism by which the alternative $\mathrm{pA}$ sites reside in the upstream regions of genes. In particular, the less frequent intronic APA involves the recognition of a cryptic intronic $\operatorname{poly}(A)$ signal that involves premature polyadenylation within the coding region affecting the sequence of the protein (Elkon et al., 2013).

Ascidians (subphylum: Tunicata) occupy a key phylogenetic position in chordate evolution and are considered the sister group of vertebrates (Delsuc et al., 2006; Swalla et al., 2000; Tsagkogeorga et al., 2009). They are proto-chordates with an innate immune system, including inflammatory humoral and cellular responses. An inflammatory response induced by LPS injection in the body wall of $C$. intestinalis is a well-established model for analysing regulator and effector inducible host defence molecules of the innate immune system, as shown for a type IX collagen-like (Vizzini et al., 2008), CAP-like (Bonura et al., 2010), MBL-like (Bonura et al., 2009), TNF $\alpha$ like (Parrinello et al., 2008; Parrinello et al., 2010), galectin-like (Vizzini et al., 2012), peroxinectin (Vizzini et al., 2013a), Interleukins 17 (Vizzini et al., 2015a), the proPO-system (Cammarata et al., 2008; Vizzini et al., 2015b) and TGF- $\beta$ (Vizzini et al., 2016). In addition, our group described the first LPS-induced APA mechanism in this species with an intronic polyadenylation signal within the first intron leading to the upregulation of a short variant (Ci8 short) of an annotated mRNA and the modification of the tissue localization of this transcript (Vizzini et al., 2013a). Following this line of evidence, we decided to pursue our analysis investigating the existence of novel APA events induced by LPS injection in the body wall of $C$. intestinalis. For this reason, we decided to analyse the expression pattern of a previously isolated CAP protein (Bonura et al., 2010) in C. intestinalis. The CAP superfamily represents a large group of proteins in several species, ranging from the yeast $S$. cerevisiae to mammals, which are involved in several cellular processes including reproduction, development, immune functions and several pathologies including cancer, nerve damage, pancreatitis and heart failure [reviewed in (Gibbs et al., 2008)].

In this manuscript, we report the identification of a novel APA mechanism by which an intronic polyadenylation signal is activated by LPS injection, leading to a shorter CAP mRNA capable of expressing the first CAP exon plus 19 amino acid residues whose sequence is contained within the first intron of the annotated gene. Furthermore, by this mechanism, the short isoform shows a 3' UTR translational regulatory element identified in immune related genes which is not present in the previously isolated CAP isoform, suggesting an intriguing scenario in which both transcriptional and post-transcriptional control mechanisms are involved in the activation of the CiCAP gene during LPS mediated inflammatory response in C. intestinalis.

\section{Materials and methods}

\subsection{Tunicates and LPS injection}

Ascidians were collected within Sciacca Harbour (Sicily, Italy), maintained in tanks with aerated sea water at $15^{\circ} \mathrm{C}$, and fed every second day with a marine invertebrate diet of coraliquid (Sera Heinsberg, Germany). Lipopolysaccharide (LPS) (Escherichia coli 055:B5, Sigma-Aldrich, Germany) solution was prepared in sterile sea water $\left(12 \mathrm{mM} \mathrm{CaCl}_{2}, 11 \mathrm{mM} \mathrm{KCl,} 26 \mathrm{mM} \mathrm{MgCl}_{2}, 43 \mathrm{mM}\right.$ Tris $\mathrm{HCl}$, $0.4 \mathrm{M} \mathrm{NaCl}, \mathrm{pH} 8.0)$. LPS solution ( $100 \mu \mathrm{g}$ LPS in $100 \mu \mathrm{l}$ sea water per animal) was injected into the tunic tissue at the median body region. Untreated ascidians were used as controls.

\subsection{Total RNA extraction}

Ascidian tissue fragments ( $200 \mathrm{mg}$ ) explanted at various times (from 1 to $48 \mathrm{~h}$ ) were immediately soaked in RNAlater Tissue collection (Ambion, Austin, TX), and stored at $-80^{\circ} \mathrm{C}$. Total RNA extraction was performed by using an RNAqueous ${ }^{\mathrm{TM}}$-Midi Kit purification system (Ambion, Austin, TX). RNA quality was assessed by agarose gel electrophoresis, and RNA concentration and purity were determined by measuring absorbance at 260 and $280 \mathrm{~nm}$.

\subsection{Gene RACE strategy and CDNA isolation}

Total RNA ( $2 \mu \mathrm{g}$ ) from ascidians treated with LPS ( $4 \mathrm{~h}$ ) was used in a PCR-based Gene RACE strategy, according to the manufacturer's instructions (GeneRacer ${ }^{\mathrm{TM}}$ Kit, Invitrogen, USA).

The strategy allows an RNA oligo to be ligated to 5' decapped mRNA molecules followed by the reverse transcription of the ligated mRNAs using the GeneRacer ${ }^{\mathrm{TM}}$ oligo dT primer and the SuperScript ${ }^{\mathrm{TM}}$ III RT Module (Invitrogen, USA). To amplify alternative CiCAP cDNAs, the ATG forward primer (all the primers are reported in Table 1$)$ was used in a $3^{\prime} \mathrm{RACE}\left(94^{\circ} \mathrm{C} 1 \mathrm{~min}, 52^{\circ} \mathrm{C} 30 \mathrm{~s}\right.$, $72^{\circ} \mathrm{C} 1 \mathrm{~min}$ for 35 cycles) paired with the GeneRacer ${ }^{\mathrm{TM}} 3^{\prime}$ primer. These reactions were diluted to $1: 100$, and nested PCRs were performed using the Exon 1 forward primer and the GeneRacer ${ }^{\mathrm{TM}}$ $3^{\prime}$ Nested primer. This reaction yielded a fragment of $638 \mathrm{bp}$ which differed from the expected CiCAP molecular weights (1392 bp); the $638 \mathrm{bp}$ amplification product was purified, cloned in PJET 1.2 blunt vector (CloneJET PCR Cloning Kit, ThermoFisher Scientific, USA) and sequenced. The $5^{\prime}$ RACE PCR $\left(94^{\circ} \mathrm{C} 1 \mathrm{~min}, 52^{\circ} \mathrm{C} 30 \mathrm{~s}, 72{ }^{\circ} \mathrm{C} 1 \mathrm{~min}\right.$ for 30 cycles) was performed with the GeneRacer ${ }^{\mathrm{TM}} 5^{\prime}$ primer and the Intron 1 Reverse primer; the reaction was diluted to 1:100 and a hemi-nested PCR was performed using the same Intron 1 Reverse primer and the GeneRacer ${ }^{\mathrm{TM}} 5^{\prime}$ Nested primer. The product was purified, cloned in pJet 1.2 blunt and sequenced.

\subsection{Sequence analysis}

Sequence similarity searches were performed using the FASTA (http://www.ebi.ac.uk/Tools/sss/fasta/nucleotide.html) and BLAST (http://www.ebi.ac.uk/Tools/sss/ncbiblast/nucleotide.html) nucleotide programs. The deduced amino acid sequence was obtained using the Expasy translation tool (http://web.expasy.org/ translate/) and a databank homology search was conducted using the BLAST protein tool (http://web.expasy.org/blast/). The presence of conserved domains was evaluated with the Delta Blast program. Alignments between the CiCAP and the CiCAP-2 proteins were accomplished with the Clustal Omega program (http://www.ebi. ac.uk/Tools/msa/clustalo/). A signal peptide in the CiCAP-2 protein was identified using the Signal IP algorithm (http://www.cbs.dtu. $\mathrm{dk} /$ services/SignalP). The Antimicrobial Peptide Database (http:// aps.unmc.edu/AP/main.php) was used to analyze the amino acid sequence of the mature CiCAP-2. To characterize the 3'UTR region of CiCAP-2, a computational analysis was performed using the Regulatory RNA Motifs and Elements Finder tool (http://regrna.mbc. nctu.edu.tw/html/prediction.html), and the mfold algorithm was employed to build up the two dimensional structure of the GAIT domain (http://unafold.rna.albany.edu/?q=mfold). 
Table 1

Primers used in CiCAP-2 study.

\begin{tabular}{|c|c|c|c|}
\hline Oligonucleotide & Sequence & $\mathrm{TM}\left({ }^{\circ} \mathrm{C}\right)$ & Lenght (nt) \\
\hline RNA oligo $^{\mathrm{TM}}$ & 5'-CGACUGGAGCACGAGGACACUGACAUGGAACUGAAGGAGUAGAAA-3' & - & 45 \\
\hline GeneRacer $^{\mathrm{TM}}$ oligo dT primer & 5'-GCTGTCAACGATACGCTACGTAACGGCATGACAGTG(T) $)_{18}-3^{\prime}$ & - & 54 \\
\hline ATG forward & 5'-ATGGATTCTAAACTGCATGT-3' & 52 & 20 \\
\hline Exon 1 forward & 5'-CAAATGCTATAGAATGGTCCGAGTCC-3' & 58 & 26 \\
\hline GeneRacer ${ }^{\mathrm{TM}}$ 3'primer & 5'-CGACTGGAGCACGAGGACACTGA-3' & 76 & 23 \\
\hline GeneRacer ${ }^{\mathrm{TM}} 3^{\prime}$ Nested primer & 5'-CGCTACGTAACGGCATGACAGTG-3' & 72 & 23 \\
\hline Intron 1 Reverse primer/3'UTR CAP-2 reverse & 5'-ATGCGTTGTATCGTCCAAGTACTCCA-3' & 59 & 19 \\
\hline GeneRacer ${ }^{\mathrm{TM}} 5$ 'primer & 5'-CGACTGGAGCACGAGGACACTGA-3' & 74 & 21 \\
\hline GeneRacer ${ }^{\mathrm{TM}} 5^{\prime}$ Nested primer & 5'-GGACACTGACATGGACTGAAGGAGTA-3' & 78 & 19 \\
\hline 3'UTR CAP-2 forward & 5'-TTGCGTGCATTGCGTTATTTTA-3' & 54 & 22 \\
\hline qRT CAP-2 forward & 5'-GTTGTTTCTTATAAATCATATACGCACCAA-3' & 56 & 30 \\
\hline qRT CAP-2 reverse & 5'- GCACGCAAAACCGAAAGAGA-3' & 55 & 20 \\
\hline CAP forward & 5'- GTATCTCCGCGAAGAGTTGG-3' & 54 & 20 \\
\hline CAP reverse & 5'-TCGGTATAACGTCGCCTCT-3' & 58 & 19 \\
\hline actin forward & 5'-TGATGTTGCCGCACTCGTA-3' & 54 & 19 \\
\hline actin reverse & 5'-TCGACAATGGATCCGGT-3' & 49 & 17 \\
\hline
\end{tabular}

\subsection{Real time $P C R$}

The tissue expression of the CiCAP and CiCAP-2 genes were examined by Real-Time PCR analysis with the Sybr-Green method (Applied Biosystems 7500 real-time PCR system), as previously described (Vizzini et al., 2013b). Primers were designed using the Custom Primers OligoPerfect Designers software (https://tools. invitrogen.com/) and synthesized commercially (Eurofins MWG Operon, Ebersberg, Germany). Tissue expression was performed in a $25 \mu \mathrm{l}$ PCR containing $2 \mu \mathrm{l}$ cDNA converted from 250 ng total RNA, $300 \mathrm{nM}$ of each forward and reverse primers (qRTCAP-2 forward and reverse for CiCAP-2 and CAP forward and reverse for the CiCAP, respectively), $300 \mathrm{nM}$ actin forward and actin reverse primers ( $C$. intestinalis actin, accession number AJ297725), and $12.5 \mu$ l Power Sybr-Green PCR Master Mix (Applied Biosystems) were used. The 50 cycles of the two-steps PCR program consisted of initial polymerase activation for $3 \mathrm{~min}$ at $95^{\circ} \mathrm{C}$ followed by a denaturing step at $95^{\circ} \mathrm{C}$ for $15 \mathrm{~s}$, and then annealing/extension was carried out at $60^{\circ} \mathrm{C}$ for $45 \mathrm{~s}$ when the fluorescent signal was detected. Each set of samples was run three times, and each plate contained quadruplicate cDNA samples and negative controls. Amplification specificity was tested by Real-Time PCR melting analysis. To obtain sample quantification, the 2DDCt method was used, and the relative changes in gene expression were analysed as described in the Applied Biosystems Use Bulletin N.2 (P/N 4303859). The amount of the CiCAP and the CiCAP-2 transcripts from the various tissues were normalized to actin in order to compensate for variations in input RNA amounts. Relative CiCAP and CiCAP-2 expressions were determined by dividing the normalized value of the target gene in each tissue sample by the normalized value obtained from the untreated tissue.

\subsection{Pharynx explant preparation and histology}

The tunic surface was cleaned and sterilized with ethyl alcohol, and pharynx fragments $(200 \mathrm{mg})$ were excised from the injection site of sham and LPS challenge ascidians. For in situ hybridization studies, pharynx fragments were fixed in Bouin's fluid (saturated picric acid:formaldehyde:acetic acid 15:5:1) for $24 \mathrm{~h}$, paraffin embedded, and serially cut at $6 \mathrm{~mm}$ (Leica RM2035 microtome, Solms, Germany).

\subsection{In situ hybridization assay (ISH)}

To examine gene expression in tissue sections, ISH was carried out with digoxigenin-11-UTP-labeled riboprobes $(1 \mu \mathrm{g} / \mathrm{ml}$ final concentration). The CiCAP probe was prepared as previously described (Bonura et al., 2010). The CiCAP-2 probe was generated by PCR amplification of a cDNA fragment of $212 \mathrm{bp}$ covering the region from nucleotide 438 to nucleotide 650 of the isolated cDNA using the 3'UTR CiCAP-2 forward and reverse primers. The digoxigenin-11-UTP-labeled riboprobes was prepared according to the manufacturer's instructions (Roche Diagnostics). The re-hydrated histological sections were digested with proteinase $\mathrm{K}(10 \mu \mathrm{g} / \mathrm{ml})$ in PBS for $5 \mathrm{~min}$, washed with PBS-T, and treated for hybridization with $50 \%$ formamide, 5X SSC (1XSSC: $0.15 \mathrm{M}$ $\mathrm{NaCl} / 0.015 \mathrm{M}$ sodium citrate, $\mathrm{pH} 7), 50 \mu \mathrm{g} / \mathrm{ml}$ heparin, $500 \mu \mathrm{g} / \mathrm{ml}$ yeast tRNA, and $0.1 \%$ Tween 20 , at $37^{\circ} \mathrm{C}$ overnight. After exhaustive washing in PBS-T and 4XSSC (twice for $10 \mathrm{~min}$ ), the sections were incubated for $1 \mathrm{~h}$ with anti-DIG-Fab-AP conjugate (Roche Diagnostics) diluted to 1:500 and washed in PBS-T. Furthermore, specimen were incubated for $1 \mathrm{~h}$ with $1 \mathrm{mM}$ levamisole in PBS-T. Finally, the sections were incubated in the 5-bromo-4-chloro-3indolyl phosphate/nitro blue tetrazolium liquid substrate system (Sigma-Aldrich,Germany). Colour development was stopped after $30 \mathrm{~min}$ at room temperature.

\subsection{Statistical methods}

Multiple comparisons were performed with one-way analysis of variance (ANOVA), and different groups were compared using Tukey's test. Standard deviations were calculated for three experiments. Values were considered statistically significant when $\mathrm{p}<0.05$. Asterisks reported within figures indicate: ${ }^{*} \mathrm{p}<0.05$; ${ }^{* *} \mathrm{p}<0.01 ;{ }^{* * *} \mathrm{p}<0.001$.

\section{Results}

\subsection{Identification of the CiCAP-2 mRNA}

By means of 3'Gene RACE technology using the Exon 1 forward primer (Table 1) and the $3^{\prime}$ Nested GeneRacer ${ }^{\mathrm{TM}}$ reverse primer (Table 1), two PCR fragments with different molecular weights were obtained. The first one was a 1392 bp long fragment with a size corresponding to the previously cloned CiCAP cDNA, while the second one was $638 \mathrm{bp}$ long (data not shown). The smaller PCR product was purified and cloned. Sequence analysis showed a common region with the published CiCAP cDNA corresponding to the first exonic sequence. The $5^{\prime}$ UTR of the shorter cDNA was isolated by $5^{\prime}$ Gene RACE using the intron 1 reverse primer and the $5^{\prime}$ Nested GeneRacer ${ }^{\mathrm{TM}}$ forward primer (Table 1 ). A fragment of $651 \mathrm{bp}$ was identified and sequenced (data not shown). The overlap of the $5^{\prime}$ and $3^{\prime}$ RACE sequences allowed us to define a full-length 


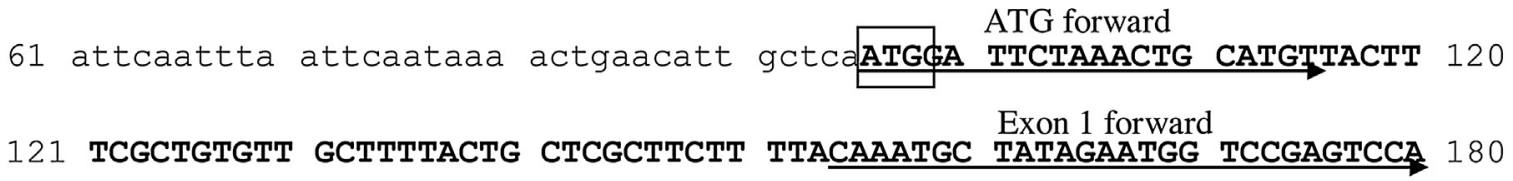

181 TAAATGCTGA AgGTAACTTA TTGTTTTTAA ATAATCAACT TTATTATAAA ATGATCAGCT 240

241 TTATTCGG AA Aataacaa ttttctaact tagttattga agctacgaac tgtacatgac 300

301 tgaaataaac cgtatagaca gatatacatt tcaattttct gacaggggtt ttacttagcg 360

qRT CAP-2 forward
361 gggggcgttg tttcttataa atcatatacg caccaacgca cgatatttgt tagagtgaaa 420

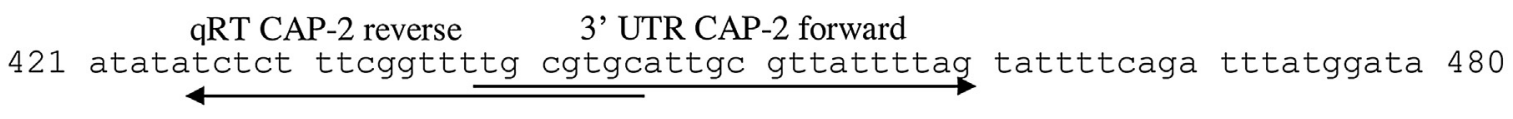

481 ctttgtttca aacgatttta aataaagg aattgcttta tgtgaacgtt gtggacgcat 540

541 tttttaaaa gacaaaaca tgctacattt aaagtttttc tacataaatg tgacccaact 600

Intron 1 Rev/3'UTR CAP-2 reverse

601 ataacatat gatgttaaat ctattggagt acttggacga tacaacgcat attttaaata 660

661 aaccactgge agatttegt caagcactat tttaggcett ttgctatgta cagcaaaaa 720

721 aaaaaaaa aa

Fig. 1. Nucleotide sequence of the full length CiCAP-2 cDNA.

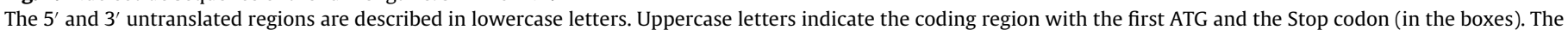

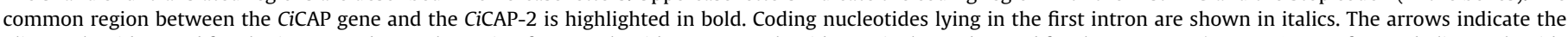

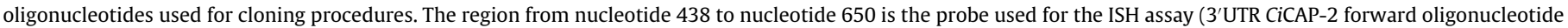
vs. 3'UTR CAP reverse oligonucleotide). The primers qRT CiCAP-2 forward and reverse were used for Real Time PCR assays.

chimeric cDNA named CiCAP-2 (Fig. 1). The complete CiCAP-2 cDNA is a 732 bp long fragment. It shows $100 \%$ identity between the 5'UTR and the first exon of the published CiCAP cDNA (region from 1 to $192 \mathrm{bp}$ ), while from 193 to $715 \mathrm{bp}$, CiCAP-2 presents $100 \%$ identity with the sequence of the first intron of the annotated CAP gene (ENSCING00000004464 localized on chromosome 8q:2,547,479-2,550,637) (see Fig. 2 for details).

\subsection{In silico analysis of the CiCAP-2 protein}

In silico analysis showed that the CiCAP-2 cDNA may codify for a putative protein of 51 amino acids with a predicted signal peptide of about 22 amino acids and a mature processed peptide of 29 amino acids. It is noteworthy that the CiCAP-2 protein was derived from the translation of a cDNA that contains the deduced coding region covering the annotated first exon plus 19 amino acid residues derived from a portion of the first intronic sequence (from amino acid 33 to 51). The schematic representations of the CiCAP-2 and CiCAP mRNAs are reported within Fig. 3 panel A. Moreover, this APA event caused the generation of a CiCAP-2 protein that lost all the conserved CAP domains and elements that we had previously found in the published CiCAP protein. Finally, amino acid analysis of the mature processed CiCAP-2 isoform showed a peculiar composition with a high total hydrophobic ratio (44\%), a relevant percentage (13\%) of isoleucine, leucine and asparagine and a total net charge of -1 . Furthermore, this peptide displayed several hydrophobic residues on the same surface, which makes it likely that this peptide may interact with membranes (Fig. 3 panel B).

\subsection{Identification of structural elements in the CiCAP-2 3'UTR}

In silico analysis of the $3^{\prime}$ UTR of the CiCAP-2 mRNA revealed the presence of several canonical signals, such as the AAUAAA polyadenylation signal (PAS), located about $250 \mathrm{nt}$ downstream from the STOP codon, flanked by an upstream sequence element (USE) (UGUA), located about $40 \mathrm{bp}$ upstream, and a CA dinucleotide and a $U$ rich downstream region (DSE), located about $30 \mathrm{bp}$ downstream from the polyadenylation signal. Both the USE and DSE have been shown to have a role in facilitating the usage of the PAS, and the strength of the PAS can be influenced by the relative positions and sequence variations of these elements (Fig. 4 panel A). All together, these elements constitute a canonical pattern that is largely conserved in all eukaryotes.

Furthermore, to identify additional cis-regulatory elements in the 3'UTR of CiCAP-2 (Fig. 2, from 252 to 732 nt), a computational analysis was performed using the REGRNA tool. Searches identified an mRNA sequence with a high degree of homology with the Interferon- $\gamma$-activated inhibitor of translation (GAIT) element, isolated for the first time into the $3^{\prime}$ UTR of the mRNA for the ceruloplasmin $(\mathrm{Cp})$ of human monocytes (Sampath et al., 
CAPgene

$\mathrm{CiCAP}-2$

CAPgene

$\mathrm{CiCAP}-2$

CAPgene

$\mathrm{CiCAP}-2$

CAPgene

$\mathrm{CiCAP}-2$

CAPgene

$\mathrm{CiCAP}-2$

CAPgene

CAPgene

CiCAP-2

CAPgene

CiCAP-2

CAPgene

CiCAP-2

CAPgene

CiCAP-2

CAPgene

CiCAP-2

CAPgene

CiCAP-2

CAPgene

CiCAP-2

CAPgene

CiCAP-2

CAPgene

CiCAP-2
CiCAP-2

agaatcctaaccacaaagttttcattaactatacaggcgaattttgctaaaaggctttct gaaatcctaaccacgaagttttcattaactatacaggcgaattttgctaaaaggctttct

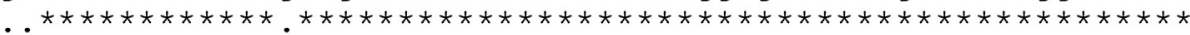

Met

attcaatttaattcaataaactgaacattgctcaATGFATTCTAAACTGCATGTTACTT

attcaatt taattcaataaactgaacattgctcaATGFATTCTAAACTGCATGTTACTT $* * * * * * * * * * * * * * * * * * * * * * * * * * * * * * * * * * * * * * * * * * * * * * * * * * * * * * * * * * * *$

TCGCTGTGTTGCTTTTACTGCTCGCTTCTTTTACAAATGCTATAGAATGGTCCGAGTCCA TCGCTGTGTTGCTTTTACTGCTCGCTTCTTTTACAAATGCTATAGAATGGTCCGAGTCCA $* * * * * * * * * * * * * * * * * * * * * * * * * * * * * * * * * * * * * * * * * * * * * * * * * * * * * * * * * * * *$

TAAATGCTGAAGgtaacttattgtttttaaataatcaactttattataaaatgatcagct TAAATGCTGAAGGTAACTTATTGTTTTTAAATAATCAACTTTATTATAAAATGATCAGCT

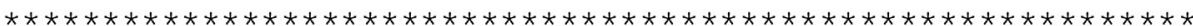
Stop ttattcggtaałaataacaattttctaacttagttattgaagctacgaactgtacatgac TTATTCGGIAA aataacaattttctaacttagttattgaagctacgaactgtacatgac

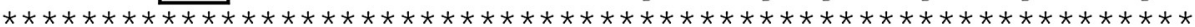

tgaaataaaccgtatagacagatatacatttcaattttctgacaggggttttacttagcg tgaaataaaccgtatagacagatatacatttcaattttctgacaggggttttacttagcg

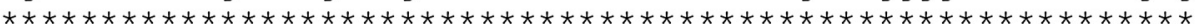

gggggcgttgtttcttataaatcatatacgcaccaacgcacgatatttgttagagtgaaa gggggcgttgtttcttataaatcatatacgcaccaacgcacgatatttgttagagtgaaa

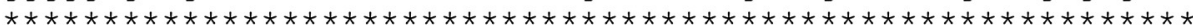

atatatctcttcggttttgcgtgcattgcgttattttagtattttcagatttatggata atatatctcttcggttttgcgtgcattgcgttattttagtattttcagatttatggata

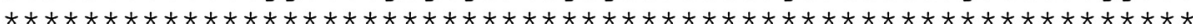

ctttgtttcaaacgattttaaaataaaaggaattgctttatgtgaacgttgtggacgca ctttgtttcaaacgattttaaaataaaaggaattgctttatgtgaacgttgtggacgca

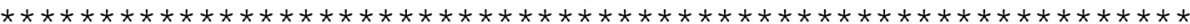

ttttttaaaagacaaaacatgctacatttaaagtttttctacataaatgtgacccaac tttttttaaagacaaaacatgctacatttaaagtttttctacataaatgtgacccaac

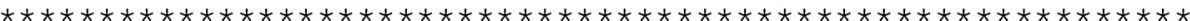

tataaacatatgatgttaaatctattggagtacttggacgatacaacgcatattttaaat tataaacatatgatgttaaatctattggagtacttggacgatacaacgcatattttaaat

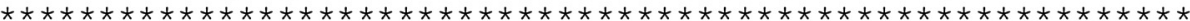

aaaccactggcagattttcgtcaagcactattttaggccttttgctatgtacagcaaaa aaaccactggcagattttcgtcaagcactattttaggcctttgctatgtacagcaaaa

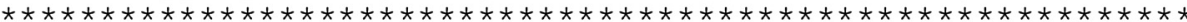

aaattcaaaacactgcttagcccaaacggaaaactgtaataatttacgtcccagtccatc

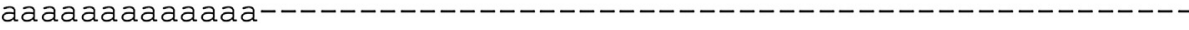
$\star \star \star:: . * * \star * . *$.

agcgttttatcattttccgtatattgtttatatatatggactgtagctggtataaattc

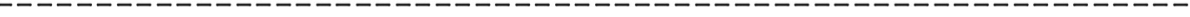

tcaaggttgcctatgttttggcag

Fig. 2. Alignment between the CiCAP-2 CDNA and the CiCAP gene annotated in the $C$. intestinalis databank.

The common region between the genomic CiCAP gene (partial sequence) and the CiCAP-2 mRNA representing the first exon is highlighted in bold. Boxes indicate the Start and Stop codons. Asterisks indicate homologies. 
A

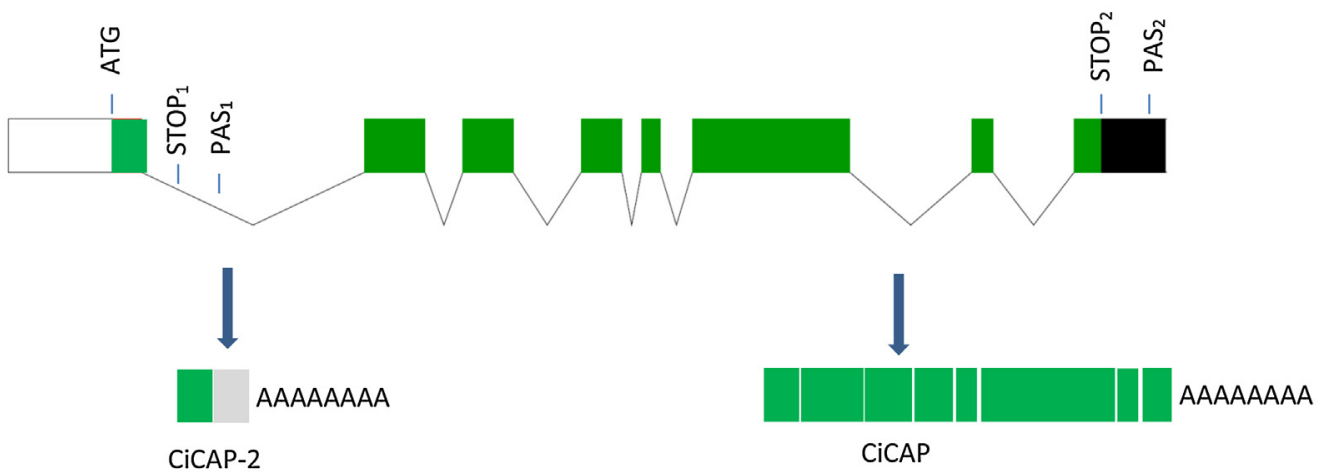

B

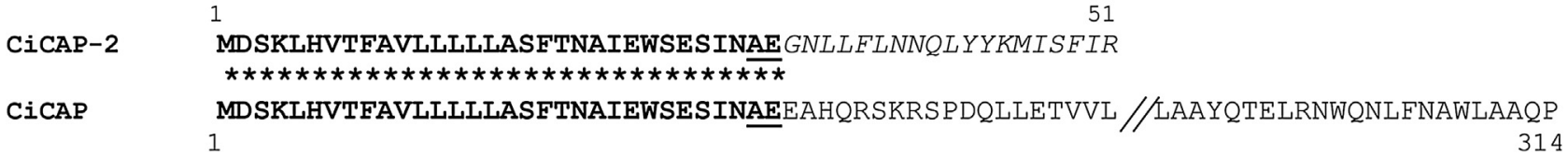

Fig. 3. Schematic representation of the two mRNA isoforms codified by the C.intestinalis CAP gene.

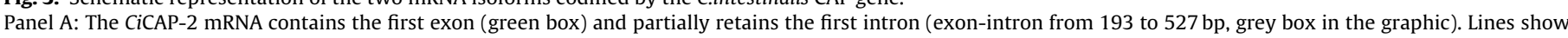

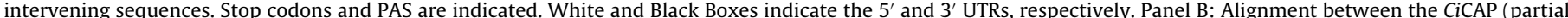

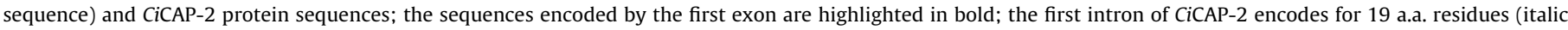
letters, from 33 to 51 ); underlined letters correspond to the putative signal peptide cut site.

2003). The CiCAP-2 GAIT element (5' UUUCUGACAGGGGUUUUACUUAGCGGGGG $3^{\prime}$ ) is located between nucleotides 336 and 365 ; it is 29 nucleotides long and exhibits the specific structural and sequence requirements of other characterized GAITs. The mfold algorithm predicted the structure of the CiCAP-2 GAIT element, showing that it contains the characteristic 6 bp proximal stem, an asymmetric bulge with a high conserved adenine in position 342 and a guanosine in position 345 (like human CCL22) (Mukhopadhyay et al., 2009). Then a structurally conserved $3 \mathrm{bp}$ distal stem with a highly conserved G-C pair and a terminal loop formed by 6 nucleotides was predicted (see Fig. 4 panels A and B for details).

\subsection{Differential expression of CiCAP-2}

To study the expression pattern of the CiCAP-2 and CiCAP mRNAs, quantitative mRNA expression in naïve, sham (inoculated with marine solution) and LPS inoculated ascidians was evaluated by Real Time PCR analysis. Four naive, sham and LPS-treated ascidians were examined at different post-inoculation time points $(1,4$, $8,12,24,48 \mathrm{~h}$ ) in three distinct experiments. Fig. 5 panels A and B display the expression of the CiCAP-2 and CiCAP mRNAs in comparison to the animals inoculated with the marine solutions showing that the CiCAP-2 variant displays a clear strong activation a few hours p.i. followed by a second wave of expression at $48 \mathrm{~h}$. On the other hand, the CiCAP mRNA was slightly enhanced at 4 p.i. only. Finally, Fig. 5 panel $C$ shows the comparison between the pattern of expression of both the CAP proteins demonstrating that the CiCAP-2 mRNA was significantly higher at $1,2,4$, and $48 \mathrm{~h}$ p.i. than the level of the CiCAP mRNA showing that LPS injection in the body wall of C.intestinalis specifically increase the expression of the CiCAP-2 variant.

\subsection{In situ hybridization assay}

Fig. 6 shows histological sections of the pharynx with vessels containing haemocytes with large granules from sham (panels $A$ and E) and LPS-treated ascidians (panels B,C,D,F,G and H) $4 \mathrm{~h}$ p.i. The CiCAP-2 localization in the pharynx from LPS-treated ascidians shows an enhanced gene expression (Fig. 6 panels $B$ to D) when compared to the sham (Fig. 6 panel A). In particular, a large number of the vessels appeared to be densely populated with haemocytes expressing the CiCAP-2 transcript (Fig. 6 panel C). The stigmata epithelium and associated haemocytes expressed CiCAP-2 mRNA after LPS activation (panel D). The CiCAP localization in the pharynx from LPS-treated ascidians shows an enhanced gene expression (Fig. 6 panels $\mathrm{F}$ to $\mathrm{H}$ ) when compared to the sham (Fig. 6 panel E), but, in agreement with Real Time PCR data, it shows a reduced expression when compared to CiCAP-2 (Fig. 6 panels $\mathrm{F}$ to $\mathrm{H}$ ). Histological sections treated with the sense strand (negative control) did not display any positive staining (CTR box within Panel A).

\section{Discussion}

The diversification of cellular functions observed in multicellular organisms may be achieved by the activation of different layers of regulatory mechanisms controlled at multiple levels, from transcription to RNA maturation and translation. Cells can increase the expression of certain genes in response to different stimuli by which transcripts and proteins may arise from alternative transcription initiation, alternative splicing, alternative polyadenylation and alternative translation initiation. This plasticity enormously expands the genome's coding capacity, modifying protein function, stability, localization and expression levels (Anderson, 2010).

For this manuscript, we analysed the pattern of expression of a previously published CiCAP gene in the attempt to identify novel 
A

253

USE

uaauaacaauuuncuaacuuaguuaungaagcuacgaaqugua faugacugaaauaaaccguauagacag

324

GAIT element

auauacauuncaaufuucugacagggguuunacuuagcggggg gcguuguuncuuauaaaucauauacgc

accaacgcacgauauuuguuagagugaaaauauaucucuuucgguuuugcgugcauugcguuauuunagu

466

polyA site

536

auuuucagaumuauggauacuunguuncaaacgauuuuaaaauaalaggaauugcuuuaugugaacguug

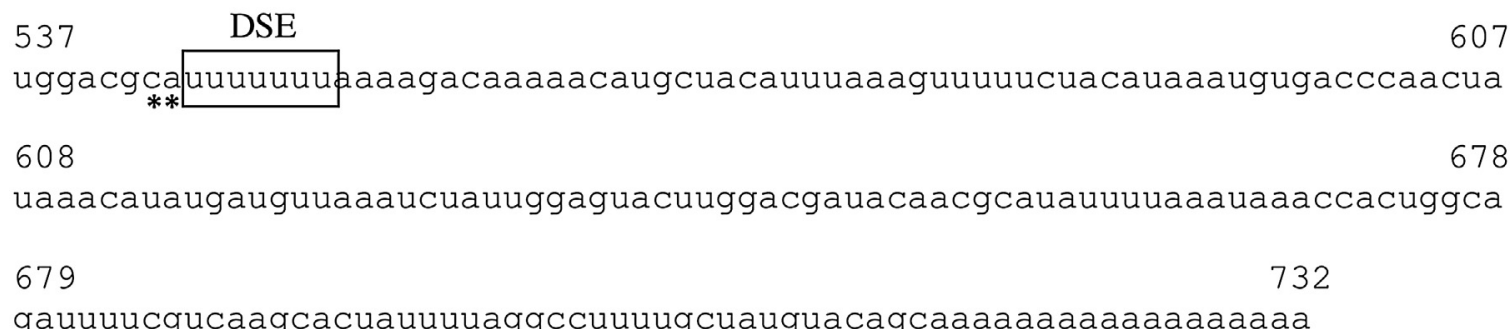

gauuucgucaagcacuauuunaggccuuungcuauguacagcaaaaaaaaaaaaaaaa

$\mathbf{B}$

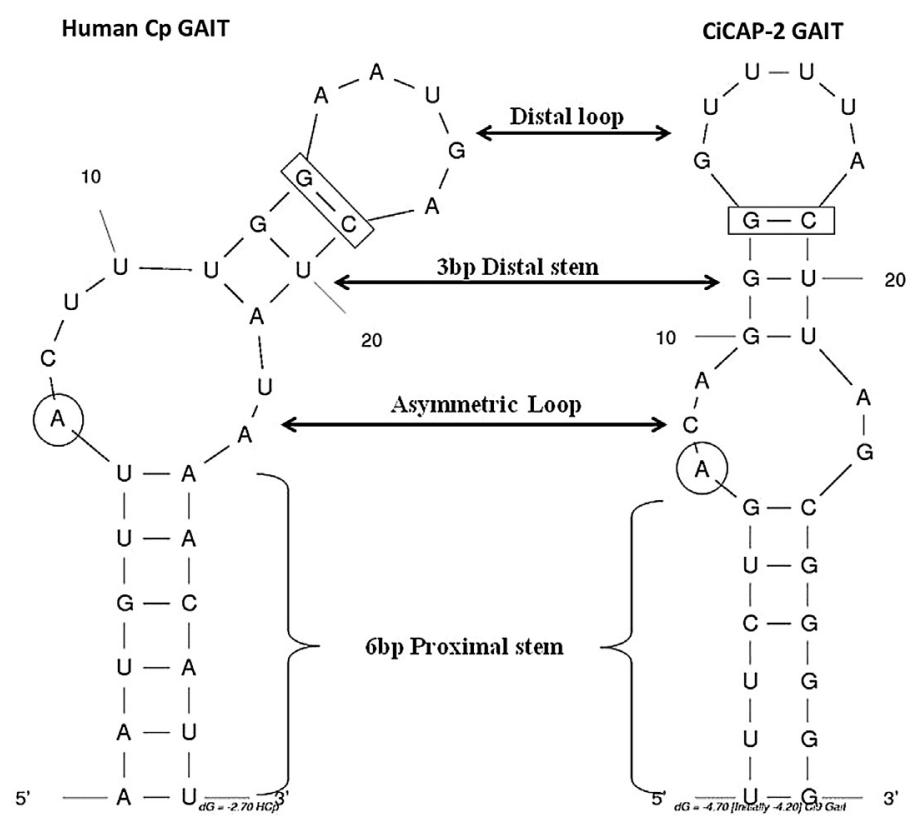

Fig. 4. Analysis of the CiCAP-2 $3^{\prime} U T R$ mRNA sequence.

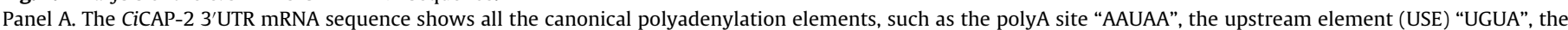

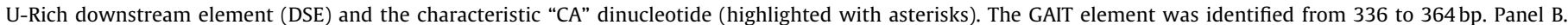

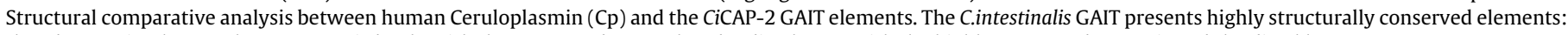
the $6 \mathrm{bp}$ proximal stem, the asymmetric bugle with the conserved A342, the 3 bp distal stem with the highly conserved G-C pair and the distal loop. 
A

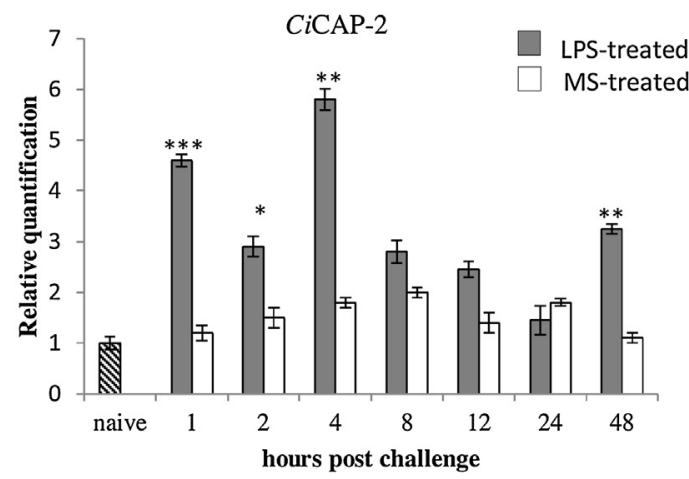

B

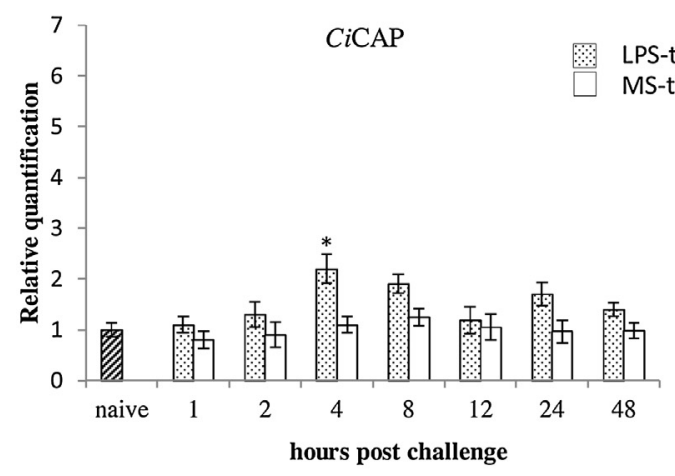

C

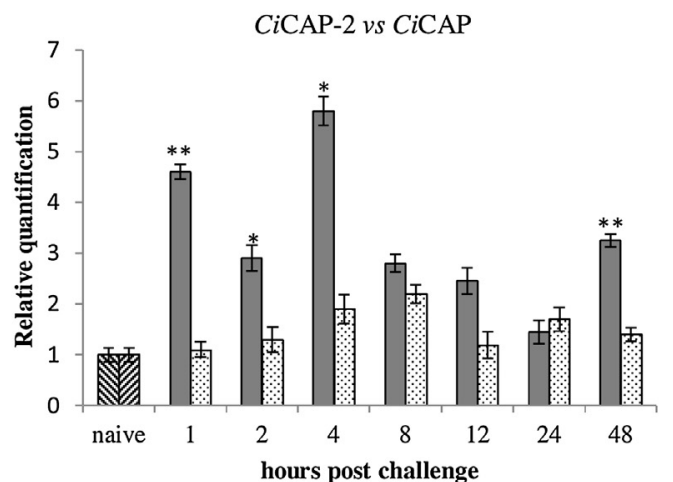

CiCAP-2

CiCAP

Fig. 5. Real-time PCR analysis.

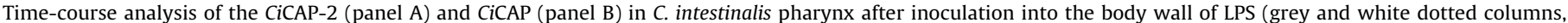

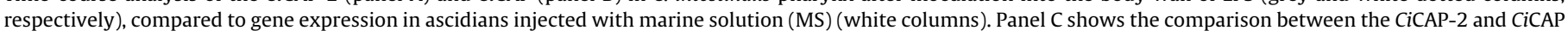

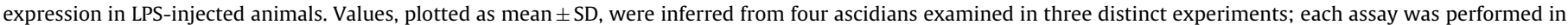
triplicate. Asterisks indicate significant differences, at each time point, between LPS and MS inoculation (post hoc Tukey $t$-test). ${ }^{*} \mathrm{P}<0.05$; ${ }^{* *} \mathrm{P}<0.01$; ${ }^{* * *} \mathrm{P}<0.001$.

regulatory mechanisms induced by LPS during the inflammatory response in C.intestinalis. In fact, CAP proteins represent a wide group of proteins that belong to the cysteine-rich secretory protein, antigen 5 and pathogenesis-related 1 superfamily which, it has been proposed, play key roles in the infection process and the modulation of immune responses in host animals (Gibbs et al., 2008).

Our data showed that a novel shorter mRNA for a CiCAP gene was generated through an APA site contained within the first intronic sequence. Alternative polyadenylation has been widely described in vertebrates, but only recently such a mechanism, affecting the expression and tissue localisation of a gene, has been described in C.intestinalis (Vizzini et al., 2013a). In this report, we describe an LPS-induced novel APA mechanism leading to the generation of an mRNA derived from the sequence of the first exon plus a stretch of nucleotides contained within the first intron with coding capacity (CiCAP-2). The possibility that intronic sequences can be maintained in the mRNA sequence has already been described in other biological systems by means of two independent mechanisms. The first one, called intron retention, describes the maintenance of an intronic sequence in the mRNA which is consequently removed by nuclear retention and exosome degradation or nonsense-mediated decay (Wong et al., 2016). In the second mechanism, introns display a coding potential ("exitrons") so that intron retention and expression affects protein features introducing novel protein domains (Staiger and Simpson, 2015). In our experimental set up, the first intron displays a dual structure with a short stretch of nucleotides with coding capacity meanwhile the remaining part represents the $3^{\prime}$ UTR region of the CiCAP-2 mRNA.
The CiCAP-2 mRNA potentially codifies for a deduced 51 amino acid long protein containing a predicted signal peptide of 22 amino acids and a 29 amino acid mature peptide that does not possess any of the previously identified CAP elements (Bonura et al., 2010), with a high hydrophobic ratio and a relevant percentage of isoleucine, leucine and asparagine.

Expression studies performed by RT-PCR in pharynx tissue showed that the CiCAP-2 mRNA is upregulated a few hours after LPS injection (1-4h), while the LPS injection procedure slightly modulated the expression levels of the CiCAP. This datum is in agreement with the in situ hybridisation, where we were able to demonstrate the co-localisation of the two mRNAs within the haemocytes flowing through the pharynx vessels and epithelium of stigmata, and the net increase of cells expressing the CiCAP-2 mRNA with respect to the cells expressing CiCAP after the LPS challenge. These analyses demonstrated that, during the LPS-induced inflammation, Ascidians reprogram the expression of a CAP protein leading to the overexpression of a short variant with structural features largely different from the original one. In addition, it is noteworthy that, during LPS-induced inflammation, flowing haemocytes present a prompt activation of the transcription of other effector and regulatory molecules, such as galectins, a proPO-system and cytokines (CiTNF, CilL-17s, CiTGF), (Parrinello et al., 2008; Parrinello et al., 2010; Vizzini et al., 2016; Vizzini et al., 2015a; Vizzini et al., 2012; Vizzini et al., 2015c; Vizzini et al., 2008) demonstrating the importance of these cells during innate immune response.

Furthermore, an in silico scan for predicted 3' UTR motifs identified the presence of a $29 \mathrm{bp}$ GAIT element located $84 \mathrm{nt}$ downstream from the STOP codon in the CiCAP-2 mRNA which was not identified 


\section{CiCap-2}
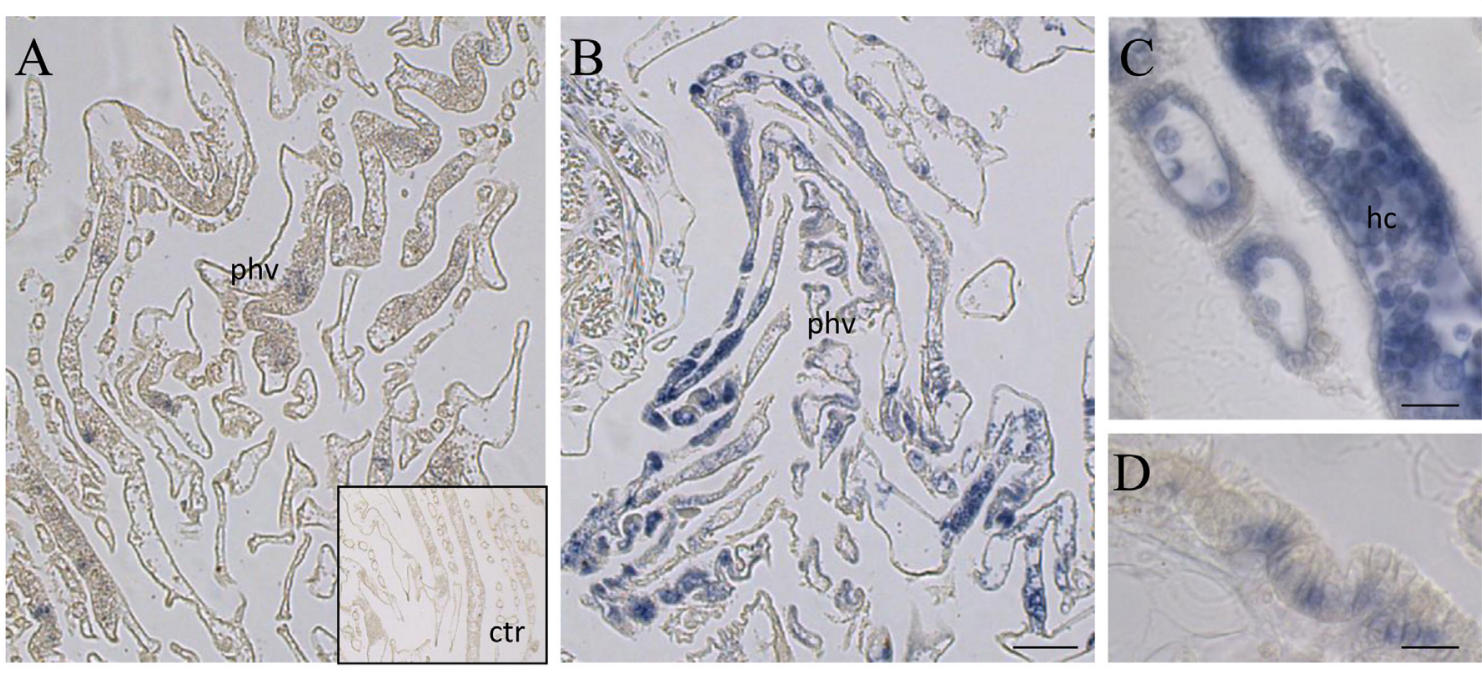

$\mathrm{CiCAP}$
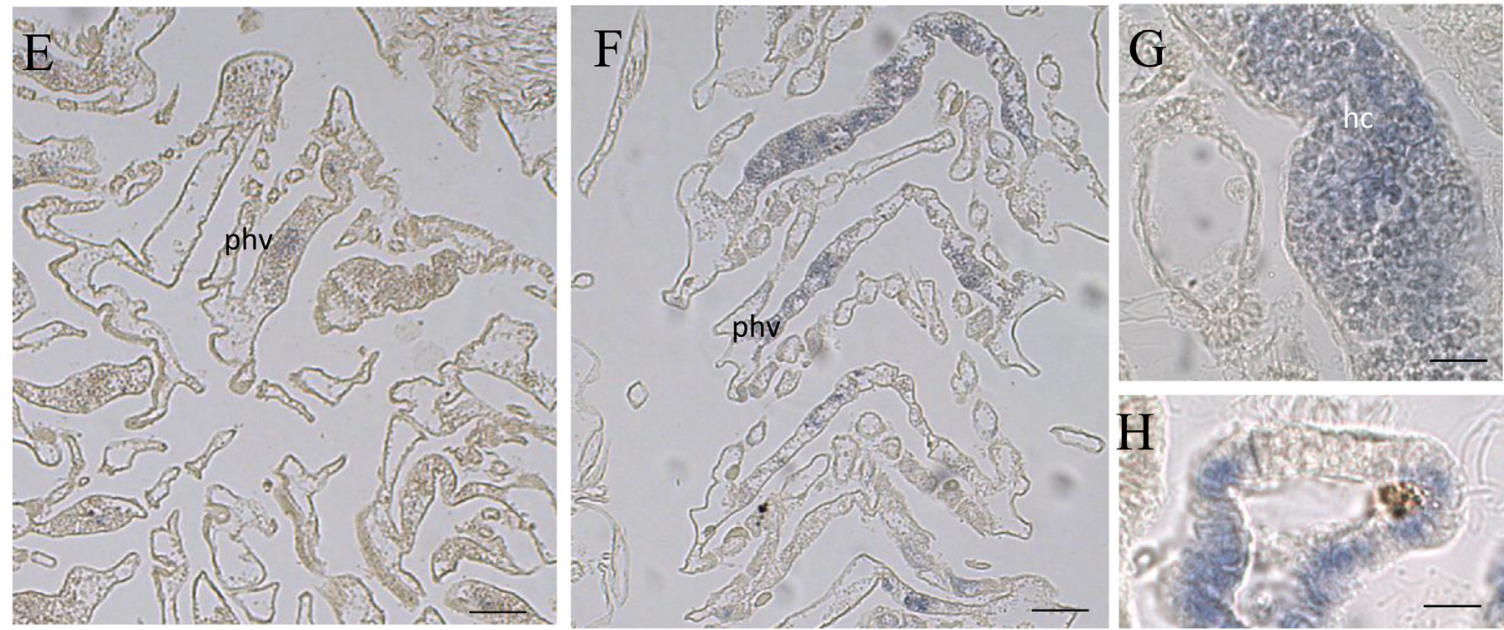

Fig. 6. Histological sections of C.intestinalis pharynx.

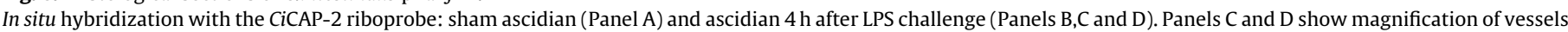

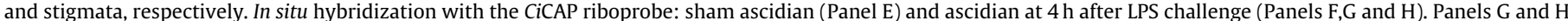

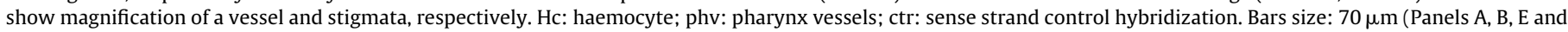
F), $25 \mu \mathrm{m}$ (Panels C and G), $10 \mu \mathrm{m}$ (Panels D and H).

in the $3^{\prime} \mathrm{UTR}$ of the CiCAP. This GAIT element is a cis-acting RNA element first identified in the 3'-UTR of the ceruloplasmin (Cp) mRNA in humans. In a more general way, GAIT elements have been found in several immune-related mRNAs showing an important role in gene-specific translation control in innate immunity (Vyas et al., 2009). These elements form a stem-loop structure that is involved in the selective translational silencing of mRNA transcripts. In invertebrates, GAIT elements have been exclusively identified in the 3'UTR of a copper zinc superoxide dismutase (CuZnSOD) gene (Sook Chung et al., 2012) and prophenoloxidase (CasPPO-hemo) (Alvarez and Chung, 2013) which are up-regulated in haemocytes after immune challenge in the blue crab Callinectes sapidus.

The GAIT complex identified in human myeloid cells is heterotetrameric, consisting of glutamyl-prolyl-tRNA synthetase (EPRS), NS1-associated protein 1 (NSAP1), ribosomal protein L13a and glyceraldehyde-3-phosphate dehydrogenase (GAPDH) (Fox, 2015). A search in the genome of C.intestinalis, showed annotated orthologue genes for EPRS, ribosomal protein L13a and GAPDH except for NSAP1. In this respect, ascidians seem to have a GAIT structure similar to the murine heterotrimeric GAIT complex, lacking NSAP1 (Arif et al., 2012).

However, whether or not the ascidian GAIT is a functional element is a matter of discussion, since no orthologue for IFN- $\gamma$ has been reported in this species, though several components of the interferon response pathway, such as the Interferon Response Factors (IRFs), have been annotated in the genome suggesting that this regulatory element might be functional in this species.

In conclusion, our manuscript describes the identification of transcriptional and post-transcriptional mechanisms of the innate immune response in C. intestinalis. In silico and in vitro studies of the expression pattern of the CiCAP gene (CiCAP-2) clearly show that ascidians are able to respond to the inflammatory injury induced by LPS with the prompt activation of a novel APA event generating the upregulation of a short isoform of a CAP mRNA that contains additional regulatory elements in the $3^{\prime}$ UTR, suggesting a tight translational control during inflammatory response.

These findings suggest that in ascidians, transcriptional and post-transcriptional regulation can rapidly activate and repress 
immune genes, controlling the initiation phase of the immune response and the resolution of the inflammation through a mechanism which has been conserved during evolution.

\section{Funding}

This work was supported by PRIN research grants from the Italian Ministry of Education (n. 20109XZEPR_003 to P.C. and n. 20109XZEPR_007 to M.C.).

\section{References}

Alvarez, J.V., Chung, J.S., 2013. Cloning of prophenoloxidase from hemocytes of the blue crab, Callinectes sapidus and its expression and enzyme activity during the molt cycle. Fish Shellfish Immunol. 35, 1349-1358.

Anderson, P., 2010. Post-transcriptional regulons coordinate the initiation and resolution of inflammation. Nat. Rev. 10, 24-35.

Arif, A., Chatterjee, P., Moodt, R.A., Fox, P.L., 2012. Heterotrimeric GAIT complex drives transcript-selective translation inhibition in murine macrophages. Mol. Cell. Biol. 32, 5046-5055.

Batra, R., Manchanda, M., Swanson, M.S., 2015. Global insights into alternative polyadenylation regulation. RNA Biol. 12, 597-602.

Bonura, A., Vizzini, A., Salerno, G., Parrinello, N., Longo, V., Colombo, P., 2009. Isolation and expression of a novel MBL-like collectin cDNA enhanced by LPS injection in the body wall of the ascidian Ciona intestinalis. Mol. Immunol. 46, 2389-2394.

Bonura, A., Vizzini, A., Salerno, G., Parrinello, D., Parrinello, N., Longo, V., Montana, G., Colombo, P., 2010. Cloning and expression of a novel component of the CAP superfamily enhanced in the inflammatory response to LPS of the ascidian Ciona intestinalis. Cell Tissue Res. 342, 411-421.

Cammarata, M., Arizza, V., Cianciolo, C., Parrinello, D., Vazzana, M., Vizzini, A., Salerno, G., Parrinello, N., 2008. The prophenoloxidase system is activated during the tunic inflammatory reaction of Ciona intestinalis. Cell Tissue Res. 333, 481-492.

Delsuc, F., Brinkmann, H., Chourrout, D., Philippe, H., 2006. Tunicates and not cephalochordates are the closest living relatives of vertebrates. Nature 439, 965-968.

Elkon, R., Ugalde, A.P., Agami, R., 2013. Alternative cleavage and polyadenylation: extent, regulation and function. Nat. Rev. Genet. 14, 496-506.

Fox, P.L., 2015. Discovery and investigation of the GAIT translational control system. RNA 21, 615-618.

Gibbs, G.M., Roelants, K., O’Bryan, M.K., 2008. The CAP superfamily: cysteine-rich secretory proteins, antigen 5 , and pathogenesis-related 1 proteins-roles in reproduction, cancer, and immune defense. Endocr. Rev. 29, 865-897.

Mukhopadhyay, R., Jia, J., Arif, A., Ray, P.S., Fox, P.L., 2009. The GAIT system: a gatekeeper of inflammatory gene expression. Trends Biochem. Sci. 34, 324-331.

Parrinello, N., Vizzini, A., Arizza, V., Salerno, G., Parrinello, D., Cammarata, M., Giaramita, F.T., Vazzana, M., 2008. Enhanced expression of a cloned and sequenced Ciona intestinalis TNFalpha-like (CiTNF alpha) gene during the LPS-induced inflammatory response. Cell Tissue Res. 334, 305-317.

Parrinello, N., Vizzini, A., Salerno, G., Sanfratello, M.A., Cammarata, M., Arizza, V., Vazzana, M., Parrinello, D., 2010. Inflamed adult pharynx tissues and swimming larva of Ciona intestinalis share CiTNFalpha-producing cells. Cell Tissue Res.
Sampath, P., Mazumder, B., Seshadri, V., Fox, P.L., 2003. Transcript-selective translational silencing by gamma interferon is directed by a novel structural element in the ceruloplasmin mRNA 3' untranslated region. Mol. Cell. Biol. 23, 1509-1519.

Sook Chung, J., Bachvaroff, T.R., Trant, J., Place, A., 2012. A second copper zinc superoxide dismutase (CuZnSOD) in the blue crab Callinectes sapidus: cloning and up-regulated expression in the hemocytes after immune challenge. Fish Shellfish Immunol. 32, 16-25.

Staiger, D., Simpson, G.G., 2015. Enter exitrons. Genome Biol. 16, 136.

Swalla, B.J., Cameron, C.B., Corley, L.S., Garey, J.R., 2000. Urochordates are monophyletic within the deuterostomes. Syst. Biol. 49, 52-64.

Tsagkogeorga, G., Turon, X., Hopcroft, R.R., Tilak, M.K., Feldstein, T., Shenkar, N., Loya, Y., Huchon, D., Douzery, E.J., Delsuc, F., 2009. An updated 18S rRNA phylogeny of tunicates based on mixture and secondary structure models. BMC Evol. Biol. 9, 187.

Vizzini, A., Pergolizzi, M., Vazzana, M., Salerno, G., Di Sano, C., Macaluso, P., Arizza, V., Parrinello, D., Cammarata, M., Parrinello, N., 2008. FACIT collagen (1alpha-chain) is expressed by hemocytes and epidermis during the inflammatory response of the ascidian Ciona intestinalis. Dev. Comp. Immunol. 32, 682-692.

Vizzini, A., Parrinello, D., Sanfratello, M.A., Salerno, G., Cammarata, M., Parrinello, N., 2012. Inducible galectins are expressed in the inflamed pharynx of the ascidian Ciona intestinalis. Fish Shellfish Immunol. 32, 101-109.

Vizzini, A., Bonura, A., Parrinello, D., Sanfratello, M.A., Longo, V., Colombo, P., 2013a. LPS challenge regulates gene expression and tissue localization of a Ciona intestinalis gene through an alternative polyadenylation mechanism. PLoS One 8, e63235.

Vizzini, A., Parrinello, D., Sanfratello, M.A., Mangano, V., Parrinello, N., Cammarata, M., 2013b. Ciona intestinalis peroxinectin is a novel component of the peroxidase-cyclooxygenase gene superfamily upregulated by LPS. Dev. Comp. Immunol. 41, 59-67.

Vizzini, A., Di Falco, F., Parrinello, D., Sanfratello, M.A., Mazzarella, C., Parrinello, N., Cammarata, M., 2015a. Ciona intestinalis interleukin 17-like genes expression is upregulated by LPS challenge. Dev. Comp. Immunol. 48, 129-137.

Vizzini, A., Parrinello, D., Sanfratello, M.A., Trapani, M.R., Mangano, V., Parrinello, N., Cammarata, M., 2015b. Upregulated transcription of phenoloxidase genes in the pharynx and endostyle of Ciona intestinalis in response to LPS. J. Invertebr. Pathol. 126, 6-11.

Vizzini, A., Parrinello, D., Sanfratello, M.A., Trapani, M.R., Mangano, V., Parrinello, N., Cammarata, M., 2015c. Upregulated transcription of phenoloxidase genes in the pharynx and endostyle of Ciona intestinalis in response to LPS. J. Invertebr. Pathol. 126C, 6-11.

Vizzini, A., Di Falco, F., Parrinello, D., Sanfratello, M.A., Cammarata, M., 2016. Transforming growth factor beta (CiTGF-beta) gene expression is induced in the inflammatory reaction of Ciona intestinalis. Dev. Comp. Immunol. 55 $102-110$.

Vyas, K., Chaudhuri, S., Leaman, D.W., Komar, A.A., Musiyenko, A., Barik, S., Mazumder, B., 2009. Genome-wide polysome profiling reveals an inflammation-responsive posttranscriptional operon in gamma interferon-activated monocytes. Mol. Cell. Biol. 29, 458-470.

Wong, J.J., Au, A.Y., Ritchie, W., Rasko, J.E., 2016. Intron retention in mRNA: no longer nonsense: known and putative roles of intron retention in normal and disease biology. BioEssays 38, 41-49.

de Klerk, E., t Hoen, P.A., 2015. Alternative mRNA transcription, processing, and translation: insights from RNA sequencing. Trends Genet.: TIG 31, 128-139. 